\title{
Electron Microscopic Study on Myocardial Lesions in Thiamine-deficient Rats
}

\author{
Tsuneo Suzuki \\ Department of Forensic Medicine (Prof. S. Akaishi), \\ Tohoku University School of Medicine, Sendai
}

\begin{abstract}
The author studied electron-microscopically myocardial lesions in thiaminedeficient rats. The changes in the heart muscle were decrease in electron density of mitochondrial matrix, swelling and rupture of mitochondria, reduction and derangement of cristae, mitochondrial degeneration, enlargement and destruction of cisternae of sarcoplasmic reticulum, appearance of large vacuoles and disappearance of cross striation in the myofibril.

These findings suggested that the cardiac lesions in thiamine deficiency were caused by disturbance of energy production, intracellular conduction of excitation and myofibrillar relaxation, which resulted from the changes of mitochondria and sarcoplasmic reticulum.
\end{abstract}

The relation of thiamine deficiency to cardiovascular disturbance has been confirmed, and it is generally recognized that sudden death may occur in cases of thiamine deficiency. Many investigators have dealt with this problem. ${ }^{1-4}$ But their findings do not always satisfactorily explain the reason of sudden death in thiamine deficiency.

The present study was made to investigate the effects of thiamine deficiency on the myocardial ultrastructure of rats and to demonstrate the cause of sudden death in this condition.

\section{Materials and Methods}

Thirty-seven adult Wistar-breed rats weighing 180 to $220 \mathrm{~g}$ were used. They were divided into two groups, the thiamine-deficiency group and the control one. Five control rats were fed on a semisynthetic diet of polished rice ${ }^{5}$ containing $3 \mathrm{mg}$ thiamine/kg. ${ }^{6}$ The other 32 rats were fed on the same diet without thiamine. Each rat was kept in a wooden box with raised metal screen bottom and provided with $20 \mathrm{~g}$ of the diet per day.

Experimental feeding of thiamine-deficient diet was continued for eight weeks or until death. Immediately before each feeding, the rats were weighed and the heart rates were counted on the electrocardiogram. Five rats each were sacrificed at the end of the second, fifth and eighth weeks. Nine out of 22 rats

Received for publication, November 15, 1966 . 
died within the sixth to eighth weeks. To 8 of 13 rats, surviving at the end of the eighth week, $0.1 \mathrm{mg}$ of thiamine was given daily after eight weeks' thiaminefree diet, and four of them were sacrificed each at the end of the tenth and twelveth weeks. The control rats were sacrificed at the end of the eighth and twelveth weeks.

As soon as the heart was excised, a slice of the left ventricular wall was taken and cut in cubes of 1 to $2 \mathrm{~mm}$, fixed in $1 \%$ osmic acid solution adjusted to $\mathrm{pH}$ 7.4 with veronal-acetate buffer in an icebox for about three hours, ${ }^{7}$ washed in water several times, and dehydrated in an ascending series of ethanol. The specimens were then embedded in epoxy resin. Sections with golden to silver hues were cut on a Hitachi type UM-3 ultramicrotome equipped with a glass knife. The sections were doubly stained with uranyl acetate and lead citrate, ${ }^{8}$ and examined directly under a Hitachi type HS-7 electron microscope. Initial magnifications were photographically enlarged as desired.

The hearts were also fixed in $10 \%$ formalin, and paraffin sections were examined light-microscopically with hematoxylin and eosin staining.

\section{Results}

\section{Gross observations and findings with light microscope}

\section{1) Control group}

During this experiment, food intake of the rats was normal. The animals gained in body weight and their general condition was good. The heart rates were about 400 to 500 per minute and regular. The hearts were macro- and microscopically normal.

\section{2) Thiamine-deficiency group}

In the second week of thiamine-free diet, food intake of each rat decreased, and retardation of growth became evident. The body weight began to decrease (Fig. 1). In the fifth week, they became emaciated and showed various degrees of alopecia. Between the sixth and eighth weeks, neuroparalytic symptoms and ataxia became remarkable, and the body weight still decreased. The heart rate gradually decreased after two weeks. At the end of the eighth week, the rats showed typical bradycardia of about 240 to 400 per minute (Fig. 2), and arrhythmia appeared in five cases. Nine out of 22 rats died between the sixth and eighth weeks. Eight rats, which were given $0.1 \mathrm{mg}$ of thiamine daily after eight weeks' thiamine-free diet, began to gain in body weight with improved food intake within several days (Fig. 1). In the fourth week, they recovered from neuroparalytic symptoms, alopecia, bradycardia and arrhythmia, and appeared quite normal.

The hearts of rats in the fifth to eighth weeks on a thiamine-free diet were slightly dilated and showed mild hypertrophy. Light-microscopically, some of them showed fatty degeneration, necrosis of myofibrils, disappearance of cross striations, interstitial edema and cell infiltration. These findings were not seen 


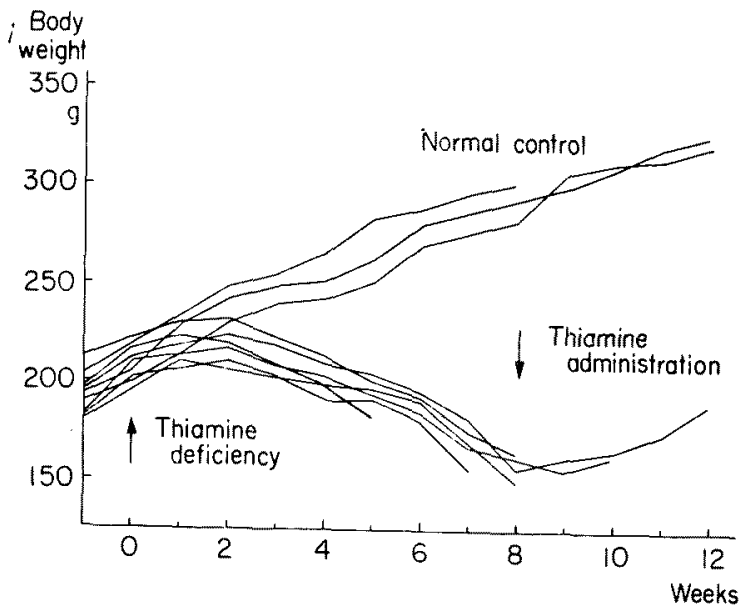

Fig. 1. Growth curves in thiamine-deficient and normal control rats.

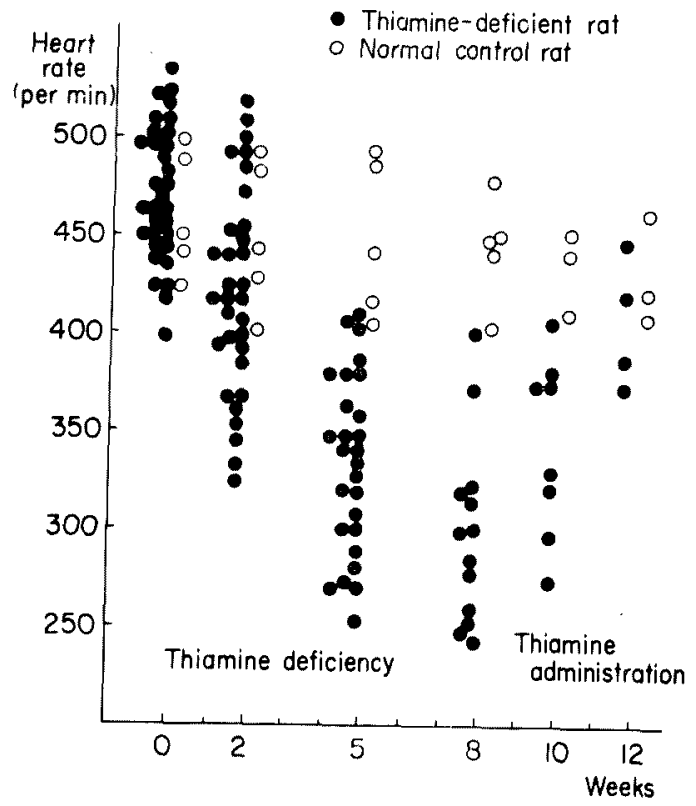

Fig. 2. Heart rates in thiamine-deficient and normal control rats.

in the animals which were given thiamine for four weeks after eight weeks' thiamine-free diet.

\section{Electron-microscopic observations}

\section{1) Control group}

The heart muscle showed the same ultrastructural appearances as those of 
the normal mammalian cardiac muscle..$^{9-11}$ Any abnormal findings attributable to the diet was not observed (Fig. 3).

\section{2) Thiamine-deficiency group}

After two weeks on a thiamine-free diet: Slight swelling of the mitochondria and reduction in the electron density of the mitochondrial matrix were observed. Neither derangement nor destruction of the cristae was found. The sarcoplasmic reticulum and myofibrils were unchanged in appearance (Fig. 4).

After five weeks on a thiamine-free diet: Most mitochondria were slightly swollen, but the external membranes were clearly demonstrated. The cristae were destroyed and reduced in number. A few mitochondria lost the greater part of their internal structure and appeared as vacuoles. The cisternae of sarcoplasmic reticulum were noticeably enlarged and increased in number. In some muscle fibers, sarcomeres of various lengths appeared (Figs. 5 and 6).

After eight weeks on a thiamine-free diet: Edematous swelling of the mitochondria was obvious. The electron density of mitochondrial matrix was reduced to such an extent that the mitochondria appeared almost transparent on electron micrograms. The majority of mitochondria were irregular in shape. The external membranes were notably uneven and some of them were ruptured. The cristae were sparse, fragmented and severely deranged. A few degenerated mitochondria and large vacuoles of about $1 \mu$ in diameter were found among the swollen and ruptured mitochondria. The cisternae of sarcoplasmic reticulum were also swollen and some of them were ruptured. Any cross striation of the myofibrils could not be distinguished. The myofibrillar columns were compressed by extremely swollen mitochondria and large vacuoles. They appeared very slender (Figs. 7-9).

After thiamine administration for two weeks following eight weeks' thiamine-free diet: The mitochondria were slightly enlarged and the electron density of mitochondrial matrix increased to a certain degree. The cristae were scanty and fragmented, but they had almost regular arrangements. A small number of large vacuoles were seen among the mitochondria. The sarcoplasmic reticulum was almost normal in shape and distribution. The cross striations of the myofibrils were barely distinguishable (Fig. 10).

After thiamine administration for four weeks following eight weeks' thiaminefree diet: The mitochondria were slightly enlarged in size and their matrices had high electron density. The cristae were well preserved and densely arranged. But a few degenerated mitochondria were present. Any large vacuoles could not be found. The sarcoplasmic reticulum showed no remarkable difference from that of the normal control rats. The cross striations of myofibrils were clearly observed (Fig. 11). 


\section{Discussion}

In the present experiments, the control normal rats did not show any abnormality. On the other hand, the rats fed on a thiamine-free diet showed a definite progress of deficiency symptom and quick recovery following the thiamine administration. It was confirmed that the diet used in this experiment met the requirement of the thiamine-free diet. However, appearance of the deficiency symptom was delayed as compared with previous reports. ${ }^{3}{ }^{12}$ The difference in days after birth was considered to be a major factor.

In 1929, Kinnersley and Peters ${ }^{13}$ discovered that thiamine was necessary for carbohydrate metabolism, and Lohmann and Schuster ${ }^{14}$ confirmed later that cocarboxylase was thiamine pyrophosphate. Because cocarboxylase is required for oxidation of pyruvate and lactate, carbohydrate oxidation is disturbed in the case of thiamine deficiency, and myocardial energy production is lowered. The heart is one of the typical organs, of which activity is sustained mainly by carbohydrate oxidation. According to Aldinger, ${ }^{15}$ progressively decreased ability of the thiamine-deficient heart muscles to generate tensile force is essentially associated with a lack of coenzyme, which, in turn, produces a defect in oxygen utilization. The disturbance of carbohydrate oxidation due to thiamine deficiency is expected to have a serious effect on the activity of the cardiac muscle.

It is conceived that thiamine is contained mainly in the liver, heart and brain, and that it tends to be transported to the heart and liver in cases of its deficient supply. Even when the animal dies of thiamine deficiency, the heart preserves more than one-fifth of its normal thiamine amount. From these facts, the heart does not naturally show any abnormality in the early stage of thiamine deficiency. Electron microscopic observations, however, revealed mitochondrial swelling and decrease in electron density of the mitochondrial matrix after two weeks' thiamine-free diet, which were followed by fragmentation of mitochondrial cristae. These changes suggested that liquid accumulated in the mitochondria. After eight weeks' thiamine-free diet, the mitochondria were extremely enlarged and the external membranes of some mitochondria were destroyed. The cristae were sparse and fragmented, and some of them showed degeneration.

Carbohydrate is said to be converted into pyruvate by enzymes in the ground sarcoplasma and then completely oxidized to carbon dioxide and water by enzymes in the mitochondria, liberating energy for biological activities. In thiamine deficiency, carbohydrate oxidation stops at the stage of pyruvate or lactate. Therefore, both pyruvate and lactate accumulate in the mitochondria. ${ }^{16}$ This causes an increase of osmotic pressure in the mitochondria, and leads to rapid inflow of large volume of liquid into the structures and results often in their rupture. The large vacuoles are brought about by the liquid and intermediate metabolic products which flow out of the destroyed mitochondria. 
At the end of the fifth week on a thiamine-free diet, the enlargement of sarcoplasmic reticulum was observed. The change was considered to have resulted from the hypofunction of mitochondria. During this period, sarcomeres of various lengths appeared in the same muscle fiber. This finding indicated that the myofibrils in the same muscle fiber were partly contracting and partly relaxing. ${ }^{17}$ The sarcoplasmic reticulum is regarded to act as an excitation-conducting factor and to effect myofibrillar relaxation. ${ }^{18}$ It is conceived that various lengths of the sarcomeres are due to interrupted conduction of excitation. From this theory, it is considered that arrhythmia and bradycardia, which appeared in the fifth to eighth weeks of thiamine-free diet, were caused by the changes of sarcoplasmic reticulum. After eight weeks' thiamine-free diet, the sarcoplasmic reticulum was extremely swollen and ruptured, and any cross striation was not distinguishing in the myofibril. In this period of thiamine deficiency, the myofibrils could neither contract nor relax because of the destruction of energy supplying system (mitochondria) and the disturbance of relaxing factor (sarcoplasmic reticulum). These changes may be a major factor contributing to diminishing tolerance against stress and to sudden death in thiamine deficiency.

Most of the ultrastructural changes disappeared when thiamine was administered for four weeks following eight weeks' thiamine-free diet. However, the mitochondria were larger in size and their matrices had higher electron density than in the control normal rats. Besides, a small number of degenerated mitochondria were observed. These facts suggested that the severely affected mitochondria were still in a state of degeneration, and those with relatively slight injuries had recovered on account of thiamine administration and became hypertrophic as a compensatory process for degenerated mitochondria.

\section{Acknowledgment}

The author expresses his cordial thanks to Dr. T. Murakami, former Professor of Forensic Medicine, Tohoku University, for his helpful suggestions, and to Prof. S. Akaishi for his advices in the preparation of manuscript.

\section{References}

1) Keefer, C.S. The beriberi heart. Arch. intern. Med., 1930, 45, 1-22.

2) Blankenhorn, M.A., Vilter, C.F., Scheinker, I.M. \& Austin, R.S. Occidental beriberi heart disease. J. Amer. med. Ass., 1946, 131, 717-726.

3) Drury, A.N., Harris, L.J. \& Maudsley, C. Vitamin B deficiency in rat. Bradycardia as distinctive feature. Biochem. $J ., 1930,24,1632-1649$.

4) Hackel, D.B., Goodale, W.T. \& Kleinerman, J. Effects of thiamine deficiency on myocardial metabolism in intact dogs. Amer. Heart $J ., 1953,46,883-894$.

5) Hirose, $Y$. Studies of the influence of amino acid composition on the thiamine deficiency disease in mice. Vitamin (Jap.), 1959, 18, 116-129.

6) Shimazono, Y. Vitamin B Complex and Vitamin $B_{1}$. Nihon Igaku Zasshi Co. (Jap.), Tokyo, 1950, pp. 79-86.

7) Caulfield, J.B. Effects of varying the vehicle for $\mathrm{Os}_{4}$ in tissue fixation. $J$. biophys. biochem. Cytol., 1957, 3, 827-830. 
8) Reynolds, E.S. The use of lead citrate at high $\mathrm{pH}$ as electron-opaque stain in electron microscopy. J. Cell Biol., 1963, 17, 208-212.

9) Moore, D.H. \& Ruska, H. Electron microscope study of mammalian cardiac muscle cells. J. biophys. biochem. Cytol., 1957, 3, 261-268.

10) Kisch, B. Electron microscopic investigation of the heart of cattle. Exp. Med. Surg., 1959, 17, 1-14.

11) Kisch, B. Electron Microscopy of the Cardiovascular System. Charles C. Thomas, Springfield, 1960, pp 10-103.

12) Yoshitoshi, Y., Shibata, N. \& Yamashita, S. Experimental studies on beriberi heart. I. Cardiac lesion in thiamine deficiency rats. Jap. Herat J., 1961, 2, 42-64.

13) Kinnersley, H.W. \& Peters, R.A. Observations upon carbohydrate metabolism in birds I. The relation between the lactic acid content of the brain and the symptoms of opisthotonus in rice-fed pigeons. Biochem. J., 1929, 23, 1126-1136.

14) Lohmann, K. \& Schuster, Ph. Untersuchungen über die Cocarboxylase. Biochem. $Z$., 1937, 294, 188-214.

15) Aldinger, E.E. Effect of thiamine deficiency on potential myocardial contractility. Circulat. Res., 1965, 16, 238-243.

16) Platt, B.S. \& Lu, G.D. Studies on the metabolism of pyruvic acid in normal and vitamine $B_{1}$-deficient states. IV. The accumulation of pyruvic acid and other carbonyl compounds in beriberi and the effect of vitamin $B_{1}$. Biochem. $J ., 1942,33$, 1525-1537.

17) Gilëv, V.P. A study of myofibril sarcomere structure during contraction. J. Cell Biol., 1962, 12, 135-147.

18) Porter, K.R. The sarcoplasmic reticulum. Its present history and present status. J. biophys. biochem. Cytol., 1961, 10, Suppl., 219-226. 


$\begin{array}{lll}\text { ss, mitochondrion } & \text { cr, crista } & \text { er, sarcoplasmic reticulum } \\ \text { sl, sarcolemma } & \text { mf, myofibril } & \text { lv, large vacuole } \\ \text { z, Z band } & \mathrm{m}, \mathrm{M} \text { band } & \mathrm{sm} \text {, sarcomere }\end{array}$

cap, capillary

Scale for all electron micrographs: 1 micron

Fig. 3. Heart muscle of a control normal rat. The mitochondria lie between the myofibrillar columns. The cross striations can be distinctly distinguished. The length of sarcomeres is almost constant. $\times 13,000$.

Fig. 4. Heart muscle after two weeks' thiamine-free diet. The sarcoplasmic reticulum and the myofibrils are almost normal. The mitochondria are somewhat swollen, and the electron density of their matrices is slightly reduced. $\times 27,000$.

Fig. 5. Heart muscle after five weeks' thiamine-free diet. The cristae are fragmented in the swollen and transparent mitochondria. The sarcoplasmic reticulum is enlarged considerably. The cross striations of the myofibrils are barely observed. $\times 19,000$.

Fig. 6. Heart muscle after five weeks' thiamine-free diet. The mitochondria are swollen and the electron density of their matrices is extremely reduced. The sarcoplasmic reticulum enlarges noticeably, but any destruction cannot be observed. The lengths of the sarcomeres are various. $\times 24,000$.

Fig. 7. Heart muscle after eight weeks' thiamine-free diet. The mitochondria are extremely swollen, and their external membranes are ruptured $(\uparrow)$. The cristae are sparse and fragmented. The sarcoplasmic reticulum is swollen and some of them are ruptured. The cross striations of the myofibril are indistinct. $\times 16,000$.

Fig. 8. Heart muscle after eight weeks' thiamine-free diet. Large vacuoles are found among swollen and ruptured mitochondria. The cristae are extremely sparse. A few degenerated mitochondria $(\uparrow)$ are observed. $\times 20,000$.

Fig. 9. Heart muscle after eight weeks' thiamine-free diet. The myofibrillar columns are slender because of compression by swollen mitochondria. The cross striations

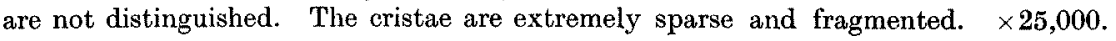

Fig. 10. Heart muscle after thiamine administration for two weeks following eight weeks' thiamine-free diet. The mitochondria are swollen but electron density of their matrices is fairly high. The cristae are still sparse and fragmented, but are almost regularly arranged. A few large vacuoles are seen among the mitochondria. The sarcoplasmic reticulum is slightly swollen. The cross striations are barely distinguished. $\times 26,000$.

Fig. 11. Heart muscle after thiamine administration for four weeks followed eight weeks' thiamine-free diet. The mitochondria are slightly swollen, but the electron density of their matrices is extremely high. A degenerated mitochondrion is noticed $(\uparrow)$. The sarcoplasmic reticulum appears almost normal. $\times 29,000$. 


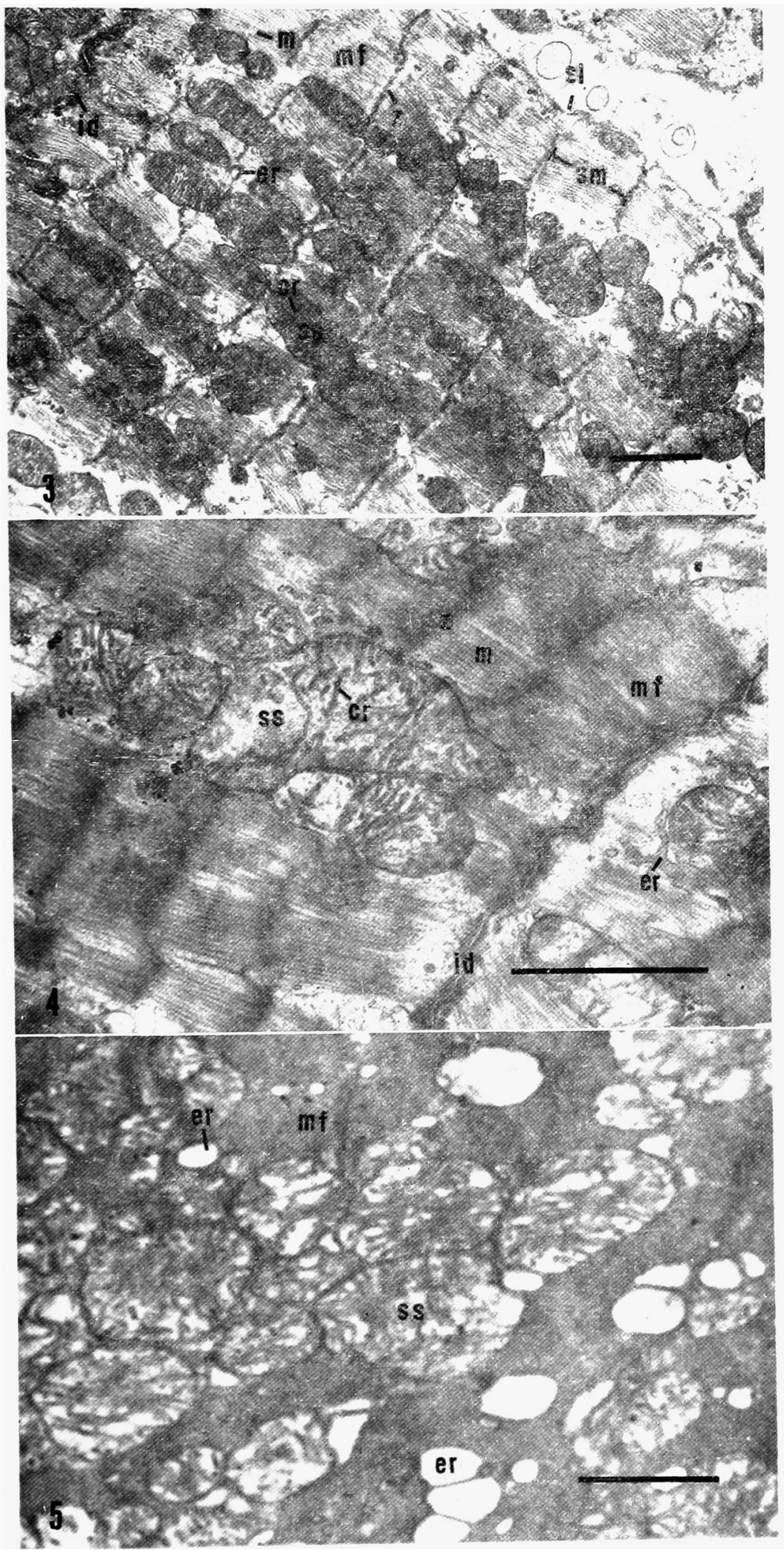




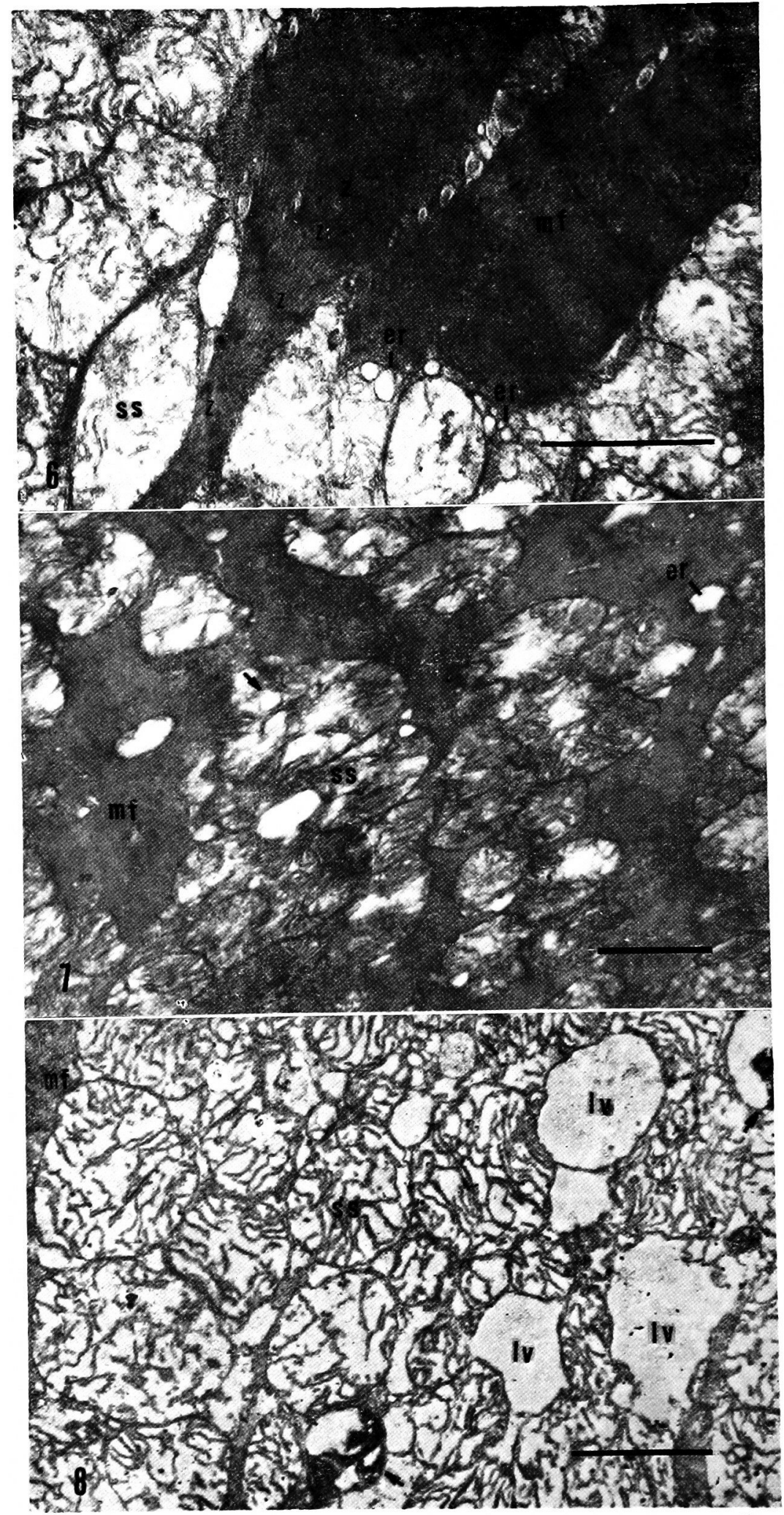




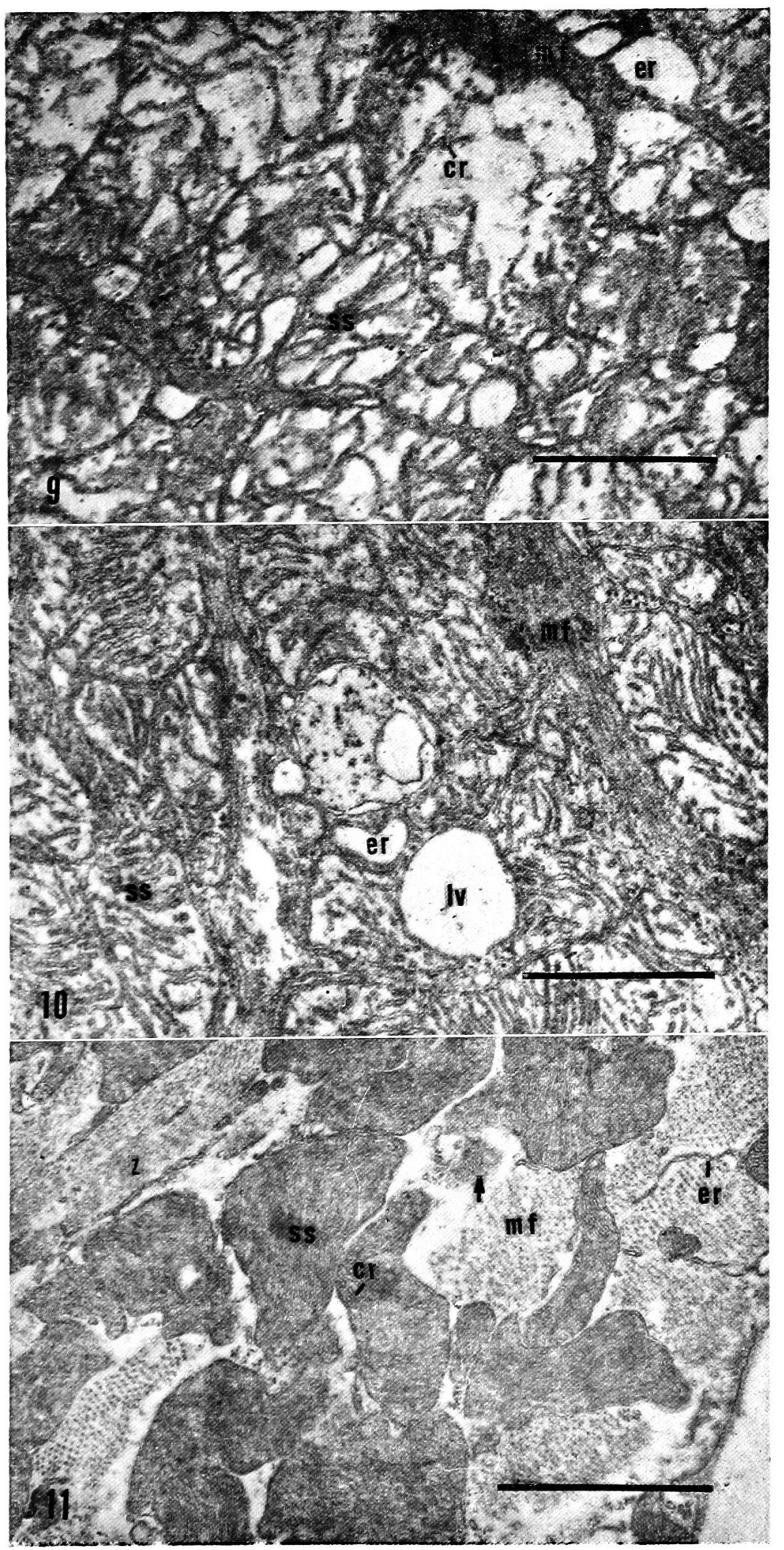

